\title{
Diagnostic utility of p63/P501S double sequential immunohistochemical staining in differentiating urothelial carcinoma from prostate carcinoma
}

\author{
Malini Srinivasan ${ }^{1}$ and Anil V Parwani $2^{2^{*}}$
}

\begin{abstract}
Background: Distinguishing urothelial carcinoma (UC) from prostate carcinoma (PC) is important due to potential therapeutic and prognostic implications. However, this can be a diagnostic challenge when there is limited tissue and in poorly differentiated tumors. We evaluated the diagnostic utility of a dual immunohistochemical stain comprising p63 and P501S (prostein), applied sequentially on a single slide and visualized by double chromogen reaction, in differentiating these two cancers. Thus far, there have been no previous studies assessing the diagnostic utility of p63 and P501S combined together as a dual immunostain in distinguishing between these two cancers.
\end{abstract}

Methods: p63/P501S dual-color sequential immunohistochemical staining was performed on archival material from 132 patients with high-grade UC and 23 patients with PC, and evaluated for p63 (brown nuclear) and P501S (red cytoplasmic) expression. Both the staining intensity and percentage of positive tumor cells were assessed.

Results: p63 was positive in 119/132 of UC and negative in PC. P501S was positive in 22/23 of PC and negative in UC. The p63+/P501S- immunoprofile had 90\% sensitivity and 100\% specificity for UC. The p63-/P501S+ immunoprofile had $96 \%$ sensitivity and 100\% specificity for PC.

Conclusion: Our results indicate that double sequential immunohistochemical staining with p63 and P501S is highly specific and can be a useful tool in distinguishing UC from PC especially when there is limited diagnostic tissue as it can be performed on a single slide.

\section{Background}

Distinction between prostate carcinoma $(\mathrm{PC})$ and urothelial carcinoma (UC) is important due to the potential therapeutic and prognostic implications. Whereas hormone therapy may be used in treatment of PC, chemotherapy is used for UC. However, discriminating between these two cancers can be a diagnostic challenge especially in high grade tumors and in the presence of limited tissue. Immunohistochemistry, using both established and newer markers, is often used as a diagnostic tool in identifying the prostatic or urothelial origin of tumors.

Among the markers used to distinguish between urothelial and prostate cancers, prostate-specific antigen

\footnotetext{
* Correspondence: parwaniav@upmc.edu

${ }^{2}$ Department of Pathology, University of Pittsburgh Medical Center,

Pittsburgh, Pennsylvania, US

Full list of author information is available at the end of the article
}

(PSA) and prostate-specific acid phosphatase (PSAP) are most commonly used to establish the prostatic origin of tumors; however, their expression is significantly decreased in poorly differentiated prostatic cancers $[1,2]$. Among the newer markers, prostate-specific membrane antigen (PSMA) and P501S (prostein) have been shown to have excellent specificity in differentiating prostate from urothelial cancers [3]. While alpha-methylacylCoA-racemase (AMACR), also known as P504S, is a useful biomarker of prostate cancer, it is also expressed in some non-prostate cancers including urothelial cancers [4] and therefore is not useful in making the distinction between PC and UC. Prostein is a prostatespecific 553 amino acid protein that was identified by cDNA subtraction in conjunction with high throughput microarray screening. It localizes to the cytoplasm, specifically to the Golgi complex, and its expression is

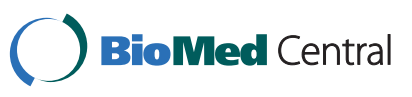


restricted to prostatic tissue and unrelated to Gleason grade $[5,6]$.

To establish urothelial differentiation, high molecular weight cytokeratin (HMWCK, clone 34ßE12), thrombomodulin, cytokeratin (CK) 7 and CK 20 are commonly used in clinical practice. However, they often have to be used as part of an antibody panel and are not specific for UC $[7,8]$. Among other markers of urothelial origin are thrombomodulin, a transmembrane glycoprotein involved in intravascular coagulation, and uroplakin III, a transmembrane protein expressed in urothelial cells. While thrombomodulin is a sensitive urothelial marker, it is also expressed in a variety of other tumors [9]. Uroplakin III is highly specific, but only moderately sensitive in identifying UC $[8,10]$. More recently p63 has emerged as a marker of urothelial differentiation [7,11]. p63 is a transcription factor belonging to the p53 family that localizes to the nucleus and shares structural and sequence homology with $\mathrm{p} 53$, and has been shown in several studies to be a marker of urothelial origin of tumors [11-13].

The aim of this study was to evaluate the diagnostic utility of p63 and P501S in distinguishing between PC and UC using dual-color immunohistochemical staining performed on a single slide by sequentially applying the antibodies.

\section{Methods}

Cases

Our study was approved by the Institutional Review Board at the University of Pittsburgh. Archival material from 139 patients with high grade UC and 25 cases with PC from the Pathology Department at the University of Pittsburgh Medical Center (UPMC) was used in this study. The UC cases consisted of 132 high grade invasive UCs and 7 high grade noninvasive UCs from radical cystectomy, radical cystoprostatectomy, or transurethral bladder resections performed at UPMC between 1992 and 2008. Routine formalin-fixed paraffin-embedded (FFPE) whole sections were used in $23 \mathrm{UC}$ cases, and the remaining 116 cases were distributed on two tissue microarrays (TMAs). The TMAs were constructed using a manual arrayer (Beecher Instruments Inc., San Prairie, WI). Two to 4 cores (core diameter $0.6 \mathrm{~mm}$ ) were represented from each urothelial cancer case. Cores from adjacent normal appearing urinary bladder tissue and other anatomic sites were also included in the TMAs. However, only the cancer containing cores were scored for this study. For prostate cancer, we used routine FFPE whole sections from 25 patients who underwent radical prostatectomy for PC at UPMC between 2000 and 2007. None of the patients received neoadjuvant chemotherapy, radiation, or hormone therapy.
Of the 139 urothelial cancer patients, 6 cases on routine sections were excluded as there was no tumor and 1 case on TMA was excluded as there was no tumor present in both the cores obtained from this patient, after immunohistochemical staining. Among the 25 patients with prostate cancer, 2 cases were excluded as there was no tumor present on the slide after performing immunohistochemistry. The remaining 132 urothelial cancer cases and 23 prostate cancer cases [Gleason score $10(n=1)$, Gleason score $9(n=6)$, Gleason score $8(\mathrm{n}=7)$, Gleason score $7(\mathrm{n}=4)$, Gleason score $6(\mathrm{n}=$ 5) were included in the final analyses.

\section{Immunohistochemistry}

Immunohistochemistry was performed on 5 micrometer sections of the FFPE routine sections and TMAs using monoclonal mouse antibodies against p63 (4A4, DAKO, Carpinteria, CA) and p501S (10E3, DAKO, Carpinteria, CA) applied sequentially. The sections were deparaffinized and hydrated, and heat induced epitope retrieval was performed using Borg decloaking high $\mathrm{pH}$ buffer in the Biocare decloaking chamber. Endogenous peroxidase activity was blocked with $3 \%$ hydrogen peroxide. The slides were first incubated with an avidin-biotin kit, followed by incubations with the p63 primary antibody (1:200 dilution) for 45 minutes, streptavidin-horseradish peroxidase, and Betazoid Diaminobenzidine for color development. Incubation with Denaturing solution for 5 minutes was done before application of the second primary antibody. P501S primary antibody (1:400 dilution) was then applied and incubated for 45 minutes, followed by incubations with alkaline phosphatase streptavidin, and Vulcan Fast Red chromogen. The slides were counterstained with Dako Hematoxylin, rinsed with water, dehydrated with alcohol and xylene, and coverslipped. Appropriate controls were included. All incubations were done at room temperature.

\section{Scoring and Evaluation}

Immunohistochemical expression was assessed semiquantitatively for staining intensity and percentage of positive tumor cells with brown nuclear staining (for p63) and red cytoplasmic staining (for P501S). Only moderate or strong staining in at least $5 \%$ of the tumor cells was considered positive. For the TMAs, a case was considered positive if at least one core showed positivity.

Immunohistochemical staining scores for p63 and P501S were individually compared between urothelial and prostate cancers, and a p-value less than 0.05 was considered statistically significant. The sensitivity, specificity, and predictive values for combined p63 and P501S immunostaining was also determined for both tumor types. 
Table 1 Immunohistochemical expression of p63 and P501S in urothelial carcinoma (UC) and prostate carcinoma (PC)

\begin{tabular}{lll}
\hline Immunohistochemical stain & $\mathbf{U C}, \mathbf{n}=\mathbf{1 3 2}(\%)$ & $\mathbf{P C}, \mathbf{n}=\mathbf{2 3}(\%)$ \\
\hline p63 & $119 / 132(90.2)$ & $0 / 23(0)$ \\
\hline p501S & $0 / 132(0)$ & $22 / 23(95.7)$ \\
\hline
\end{tabular}

\section{Results}

The immunohistochemical findings are summarized in Table 1. Neither UC nor PC was positive for both p63 and p501S. Statistically significant p-values were observed for p63 and P501S expression, individually, in the distinction of UC from PC. One hundred nineteen of 132 (90.2\%) UC were positive and none of the PC were positive for $\mathrm{p} 63(\mathrm{p}<0.0001)$. Twenty-two of 23 (95.7\%) PC cases were positive and none of the UC cases were positive for p501S ( $<<0.0001)$. p63 showed diffuse brown nuclear staining in urothelial cancers. p63 expression was also seen in the basal layer of the benign prostate glands included in some of the sections and this served as a positive internal control for p63 staining. P501S showed red granular perinuclear cytoplasmic staining of prostate cancer cells. Benign prostatic tissue included in some of the cases also showed P501S positivity (Figure 1).

The p63 and P501S combination immunohistochemical profiles in UC and PC are illustrated in Figure 2. The sensitivity, specificity, and predictive values of p63 and P501S combination immunoprofiles for distinguishing PC from UC are shown in Table 2. The p63+/p501S- immunohistochemical profile was seen only in UCs $(90.2 \%$ sensitivity, $100 \%$ specificity, and $100 \%$ positive predictive value) and the p63-/p501S+ imunohistochemical profile was seen only in PCs $(95.7 \%$ sensitivity, $100 \%$ specificity, and $100 \%$ positive predictive value).

\section{Discussion}

Morphological distinction of UC from PC can often be a diagnostic challenge especially in poorly differentiated tumors. Additionally, serum PSA levels may be raised in urothelial cancers that infiltrate the prostate gland adding to the diagnostic dilemma. Immunohistochemistry is often used as a diagnostic tool to accurately distinguish between these two tumors and establish the final diagnosis [14]. The difficulty in making an accurate distinction is further compounded when there is only limited tissue available, such as in needle biopsies, cell blocks and fine needle aspirations with only small foci of carcinoma, when additional sections may have to be ordered and there may not be adequate tissue remaining to perform multiple immunohistochemical stains on separate slides.

Although the diagnostic utility of p63 and P501S in distinguishing between primary PC and UC have been individually evaluated previously $[3,11,15]$, thus far, there have been no previous studies evaluating these two markers together either as a cocktail or applied sequentially. Our results indicate that dual-color immunohistochemistry with p63 and P501S applied

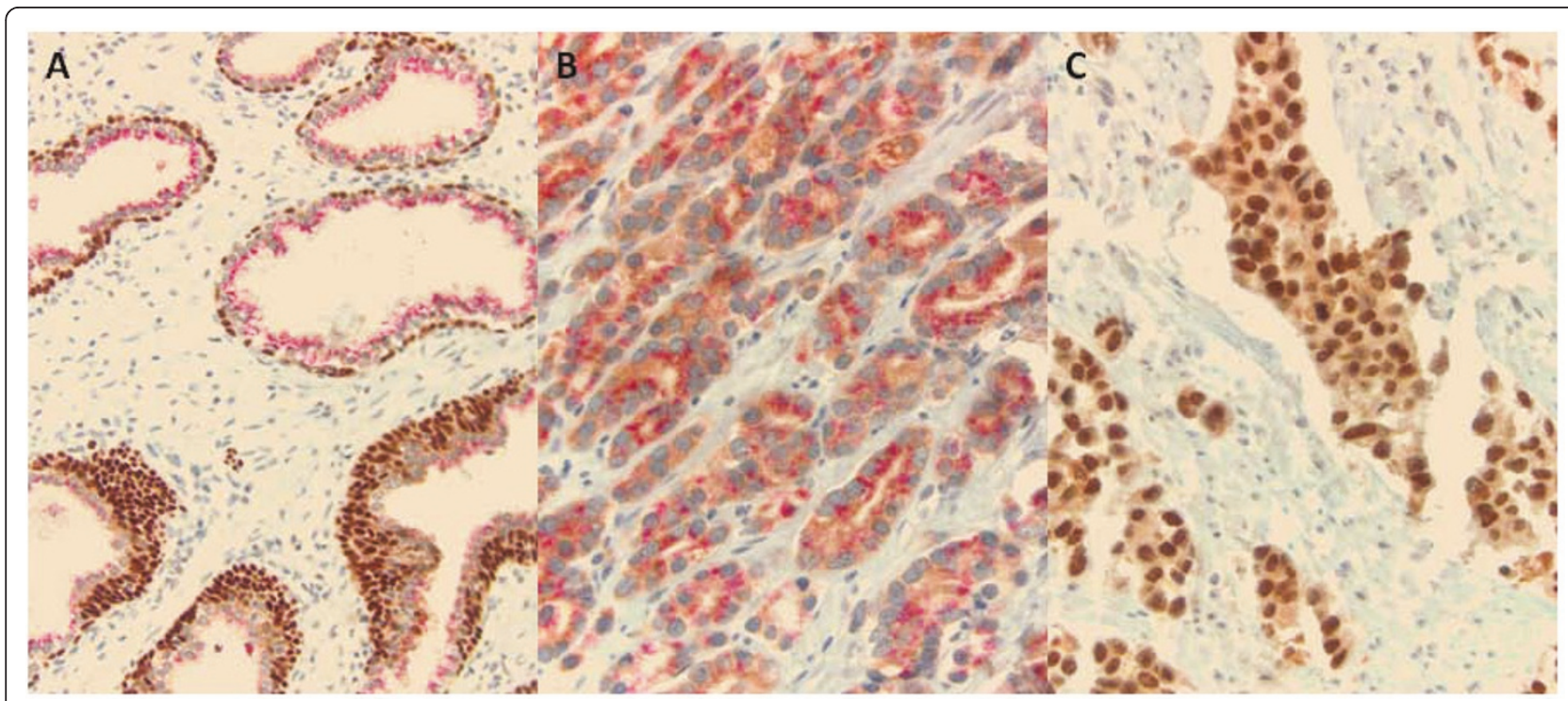

Figure 1 Dual p63/P501S immunohistochemical stain. A. Benign prostate glands with foci of basal cell hyperplasia showing red granular perinuclear cytoplasmic P501S staining and diffuse brown nuclear p63 staining of basal cell layer (X200.); B. Prostate cancer with red perinuclear cytoplasmic P501S staining and no brown nuclear p63 staining (X400); C. Urothelial carcinoma with diffuse brown nuclear p63 staining and absence of red P501S staining (X400). 


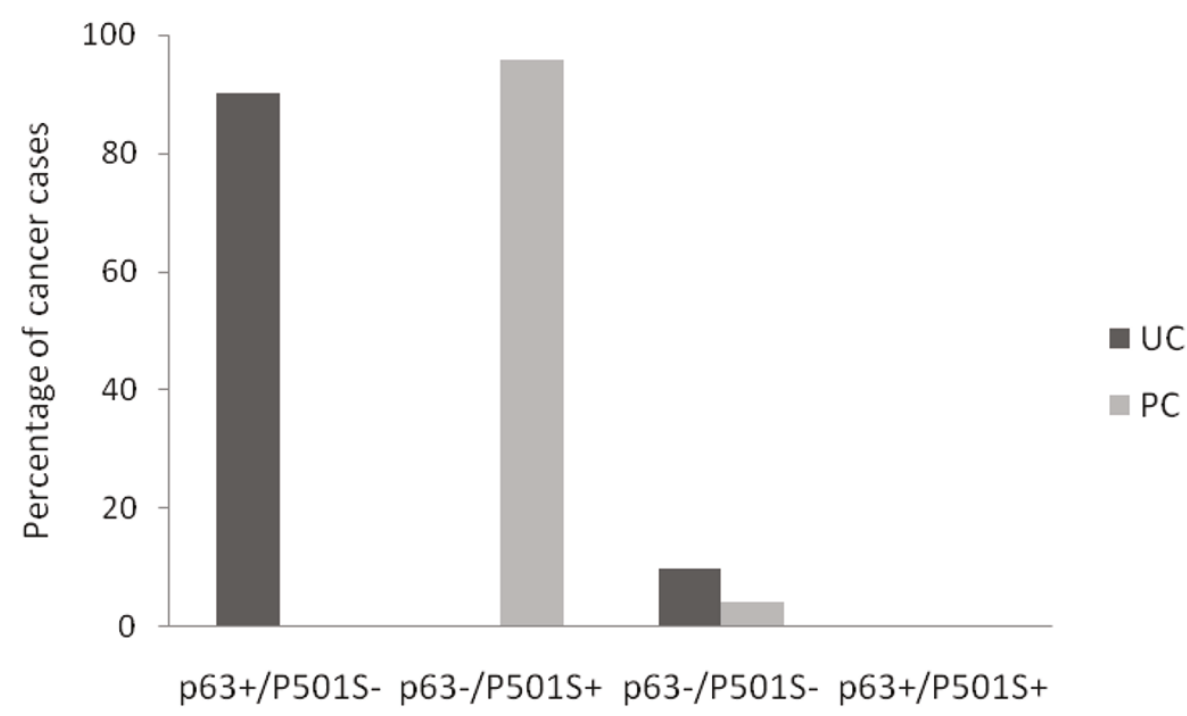

Figure 2 p63/P501S combination immunohistochemical profile in urothelial carcinoma (UC) and prostate carcinoma (PC).

sequentially shows excellent specificity for distinguishing UC from PC. The differential localization (diffuse nuclear for p63 versus granular cytoplasmic for P501S) combined with the double chromogen reaction facilitate easy visualization (brown for p63 and red for P501S) and enable quick and easy interpretation of the markers all in one slide. Additionally, this technique can be easily performed in the laboratory and conserves tissue as it is performed on a single slide. Thus, this immunostain could be a potentially valuable tool to aid in the distinction between these two cancers in the presence of limited tissue. However, in order to accurately characterize this double sequential immunostain, further studies comparing its performance with immunohistochemistry performed using the same antibodies individually as well as comparing its performance in prostate needle biopsies versus radical prostatectomies are required.

In the current study, p63 positivity was seen in 119/ 132 (90\%) of UC cases. None of the PCs were positive for $\mathrm{p} 63$. Of the $13 \mathrm{UC}$ cases that were negative for $\mathrm{p} 63$, 3 cases had micropapillary features. Our results are comparable to Kunju et al [15] who also found diffuse nuclear p63 positivity in $92 \%$ of their UC cases using the same p63 monoclonal antibody. Chuang et al [3] using the same p63 antibody found p63 in 83\% of their UC cases. Similar to our study, Kunju et al and Chuang et al did not observe nuclear p63 positivity in any of their PCs. Thus, p63 appears to be a useful marker in distinguishing between UC and PC due to its high specificity for UC.

We found granular perinuclear cytoplasmic P501S expression in 22/23 (96\%) PC cases. There was no difference in P501S staining intensity across PC cases according to Gleason scores. None of the UCs in our study showed P501S positivity. Our results are similar to Kalos et al [5] who found prostein expression in 111/ 118 (94\%) primary and metastatic prostate cancers. They also found prostein to have excellent specificity with no expression detected in 4,635 normal and malignant non-prostatic tissues. Chuang et al observed P501S positivity in all of their $38 \mathrm{PC}$ cases. These authors also found P501S to have high specificity with only $2 / 35$ (6\%) high grade UC showing focal weak positivity. Other studies $[16,17]$ have also shown prostein expression to be a highly specific marker for identification of prostatic origin of tumors. The granular perinuclear cytoplasmic expression of prostein is an important feature in establishing the prostatic origin of tumors. A recent study by Lane et al [18] found moderate diffuse cytoplasmic P501S staining in $11 \%$ of urinary bladder adenocarcinomas.

The single P501S negative case in our study was diagnosed as a poorly differentiated prostatic adenocarcinoma (Gleason score 9). The focus of carcinoma seen

Table 2 Sensitivity, specificity, and predictive values: p63/p501S immunostaining in urothelial carcinoma (UC) and prostate carcinoma (PC)

\begin{tabular}{lllll}
\hline Immunohistochemical profile & Sensitivity & Specificity & Positive predictive value & Negative predictive value \\
\hline P63+/p501S- for UC & $90.2 \%$ & $100 \%$ & 1 & 0.639 \\
P63-/P501S+ for PC & $95.7 \%$ & $100 \%$ & 1 & 0.992 \\
\hline
\end{tabular}


on the immunostain slide of this case showed atrophic features and p63 negativity for basal cells. Although prostein expression was absent in this focus of carcinoma, adjacent areas of high-grade prostatic intraepithelial neoplasias (HGPIN) expressed prostein and also showed p63 reactivity in the basal cells. It has been previously described that the expression of AMACR, is absent or decreased in atrophic PCs [19]. It is possible that prostein expression may similarly be decreased in prostate cancer with atrophic features. This could lead to a potential diagnostic pitfall especially in rare cases of prostate cancer when p63 is aberrantly expressed in a non-basal distribution [20]. Due to the potential impact on clinical practice, further studies are required to validate our finding and determine if prostein immunoreactivity varies among specific morphological variants of $\mathrm{PC}$.

Our findings indicate that p63/P501S dual immunostaining shows excellent specificity and good sensitivity in distinguishing urothelial from PC. A p63+/P501Simmunoprofile favors a diagnosis of UC with $90.2 \%$ sensitivity and $100 \%$ specificity, while a p63-/P501S+ profile establishes a diagnosis of PC with $95.7 \%$ sensitivity and $100 \%$ specificity. The p63-/P501S- profile was seen in 14 cases (9\%), 13 of which were urothelial cancers. Caution should be exercised in interpreting a p63-/P501S- profile as lack of p63expression does not rule out UC. Kunju et al [15] using a panel comprising PSA, HMWCK, and p63 to distinguish between PC and UC in 26 diagnostically challenging cases found p63 positivity in 10/13 UCs. The 3 remaining p63 negative UCs were also negative for HMWCK, and they established the urothelial origin using CK 7 and CK 20 expression. In a study by Higgins et al [11] that included 321 bladder UCs $(\mathrm{n}=$ 238 high grade, $\mathrm{n}=83$ low grade) and 267 PCs, placental S100 (S100P) and GATA 3 emerged as markers associated with urothelial differentiation, and S100P was found to have higher sensitivity for UCs. They found p63 expression in $87 \%$ of UCs and only $0.4 \%$ of PCs. However, when the expression of S100P was also considered, $94.9 \%$ of all UCs expressed one or both markers, while none of their PCs expressed both p63 and S100P. Higgins et al concluded that the expression of S100P and p63 are partly complementary and when used in combination each marker may identify UC cases missed by the other. Some studies have shown high sensitivity for detection of UC with HMWCK (clone 34ßE12) [21] and thrombomodulin [9]. Immunohistochemistry using a triple antibody combination of p63, P501S, and S100P or HMWCK or thrombomodulin may increase the sensitivity of the p63/P501S immunostain for detection of $\mathrm{UC}$, and needs to be confirmed in future studies.

\section{Conclusion}

In conclusion, our results indicate that double sequential immunostaining with $\mathrm{p} 63 / \mathrm{p} 501 \mathrm{~S}$ is highly specific in distinguishing primary $\mathrm{PC}$ from $\mathrm{UC}$, and support the potential clinical utility of this immunostain as a diagnostic tool in distinguishing between these two cancers in settings where only limited diagnostic material is available since it enables simultaneous evaluation of both markers on a single histologic slide. Using a triple immunostain by adding another sensitive urothelial marker may further improve the diagnostic performance of this immunostain.

\section{Funding}

This work was supported in part by the University of Pittsburgh Cancer Center Support Grant (CCSG) P30 CA047904 and Department of Pathology at the University of Pittsburgh Medical Center.

\section{Acknowledgements}

We thank Ms. Marie Acquafondata for technical assistance with the p63/ P501S double sequential immunohistochemical staining.

\section{Author details}

'Department of Epidemiology, Graduate School of Public Health, University of Pittsburgh Cancer Institute, Pittsburgh, Pennsylvania, US. ${ }^{2}$ Department of Pathology, University of Pittsburgh Medical Center, Pittsburgh, Pennsylvania, US.

\section{Authors' contributions}

MS was involved in the design of the study, performed statistical and immunohistochemical analysis, and drafted the manuscript. AVP conceived the study, was involved in the design and immunohistochemical analysis, and edited the manuscript for intellectual content. All authors read and approved the final manuscript.

\section{Competing interests}

The authors declare that they have no competing interests.

Received: 7 July 2011 Accepted: 21 July 2011 Published: 21 July 2011

\section{References}

1. Aihara M, Lebovitz RM, Wheeler TM, Kinner BM, Ohori M, Scardino PT: Prostate specific antigen and gleason grade: an immunohistochemical study of prostate cancer. J Urol 1994, 151:1558-1564.

2. Goldstein NS: Immunophenotypic characterization of 225 prostate adenocarcinomas with intermediate or high Gleason scores. Am J Clin Pathol 2002, 117:471-477

3. Chuang AY, DeMarzo AM, Veltri RW, Sharma RB, Bieberich CJ, Epstein Jl: Immunohistochemical differentiation of high-grade prostate carcinoma from urothelial carcinoma. Am J Surg Pathol 2007, 31:1246-1255.

4. Jiang Z, Fanger GR, Woda BA, Banner BF, Algate P, Dresser $K$, et al: Expression of alpha-methylacyl-CoA racemase (P504s) in various malignant neoplasms and normal tissues: astudy of 761 cases. Hum Pathol 2003, 34:792-796.

5. Kalos M, Askaa J, Hylander BL, Repasky EA, Cai F, Vedvick T, et al: Prostein expression is highly restricted to normal and malignant prostate tissues. Prostate 2004, 60:246-256.

6. Xu J, Kalos M, Stolk JA, Zasloff EJ, Zhang X, Houghton RL, et al: Identification and characterization of prostein, a novel prostate-specific protein. Cancer Res 2001, 61:1563-1568.

7. Hodges KB, Lopez-Beltran A, Emerson RE, Montironi R, Cheng L: Clinical utility of immunohistochemistry in the diagnoses of urinary bladder neoplasia. Appl Immunohistochem Mol Morphol 2010, 18:401-410. 
8. Parker DC, Folpe AL, Bell J, Oliva E, Young RH, Cohen C, et al: Potential utility of uroplakin III, thrombomodulin, high molecular weight cytokeratin, and cytokeratin 20 in noninvasive, invasive, and metastatic urothelial (transitional cell) carcinomas. Am J Surg Pathol 2003, 27:1-10.

9. Ordonez NG: Thrombomodulin expression in transitional cell carcinoma. Am J Clin Pathol 1998, 110:385-390.

10. Kaufmann O, Volmerig J, Dietel M: Uroplakin III is a highly specific and moderately sensitive immunohistochemical marker for primary and metastatic urothelial carcinomas. Am J Clin Pathol 2000, 113:683-687.

11. Higgins JP, Kaygusuz G, Wang L, Montgomery K, Mason V, Zhu SX, et al: Placental S100 (S100P) and GATA3: markers for transitional epithelium and urothelial carcinoma discovered by complementary DNA microarray. Am J Surg Pathol 2007, 31:673-680.

12. Di Como CJ, Urist MJ, Babayan I, Drobnjak M, Hedvat CV, Teruya-Feldstein J, et al: p63 expression profiles in human normal and tumor tissues. Clin Cancer Res 2002, 8:494-501.

13. Nekulova M, Holcakova J, Coates P, Vojtesek B: The role of p63 in cancer, stem cells and cancer stem cells. Cell Mol Biol Lett 2011, 16:296-327.

14. Varma M, Jasani B: Diagnostic utility of immunohistochemistry in morphologically difficult prostate cancer: review of current literature. Histopathology 2005, 47:1-16.

15. Kunju LP, Mehra R, Snyder M, Shah RB: Prostate-specific antigen, highmolecular-weight cytokeratin (clone 34betaE12), and/or p63: an optimal immunohistochemical panel to distinguish poorly differentiated prostate adenocarcinoma from urothelial carcinoma. Am J Clin Pathol 2006 125:675-681.

16. Sheridan T, Herawi M, Epstein Jl, Illei PB: The role of P501S and PSA in the diagnosis of metastatic adenocarcinoma of the prostate. Am J Surg Pathol 2007, 31:1351-1355.

17. Yin $M$, Dhir R, Parwani AV: Diagnostic utility of p501s (prostein) in comparison to prostate specific antigen (PSA) for the detection of metastatic prostatic adenocarcinoma. Diagn Pathol 2007, 2:41.

18. Lane Z, Hansel DE, Epstein Jl: Immunohistochemical expression of prostatic antigens in adenocarcinoma and villous adenoma of the urinary bladder. Am J Surg Pathol 2008, 32:1322-1326.

19. Farinola MA, Epstein Jl: Utility of immunohistochemistry for alphamethylacyl-CoA racemase in distinguishing atrophic prostate cancer from benign atrophy. Hum Pathol 2004, 35:1272-1278.

20. Osunkoya AO, Hansel DE, Sun X, Netto GJ, Epstein Jl: Aberrant diffuse expression of p63 in adenocarcinoma of the prostate on needle biopsy and radical prostatectomy: report of 21 cases. Am J Surg Pathol 2008, 32:461-467.

21. Varma M, Morgan M, Amin MB, Wozniak S, Jasani B: High molecular weight cytokeratin antibody (clone 34betaE12): a sensitive marker for differentiation of high-grade invasive urothelial carcinoma from prostate cancer. Histopathology 2003, 42:167-172.

doi:10.1186/1746-1596-6-67

Cite this article as: Srinivasan and Parwani: Diagnostic utility of p63/ P501S double sequential immunohistochemical staining in differentiating urothelial carcinoma from prostate carcinoma. Diagnostic Pathology 2011 6:67.

\section{Submit your next manuscript to BioMed Central and take full advantage of:}

- Convenient online submission

- Thorough peer review

- No space constraints or color figure charges

- Immediate publication on acceptance

- Inclusion in PubMed, CAS, Scopus and Google Scholar

- Research which is freely available for redistribution

Submit your manuscript at www.biomedcentral.com/submit
Ciomed Central 